\title{
On the Enhancement of LDPC Codes Used in WiMAX
}

\author{
Khaled ElMahgoub ${ }^{*, 1}$ and Mohammed Nafie*,2 \\ ${ }^{1}$ Cairo University, Dept. of Electronics and Communications, Giza, Egypt \\ ${ }^{2}$ Nile University, Wireless Intelligent Networks Center, Giza, Egypt
}

\begin{abstract}
In this paper the low density party check (LDPC) codes used in the IEEE 802.16 standard physical layer are studied, and two novel techniques to enhance the performance of such codes are introduced. In the first technique, a novel parity check matrix for LDPC codes over $G F(4)$ with the non-zero entries chosen to maximize the entropy is proposed, the parity check matrix is based on the binary parity check matrix used in the IEEE 802.16 standard. The proposed code is proven to outperform the binary code used in the IEEE 802.16 standard over both additive white Gaussian noise (AWGN) and Stanford University Interim (SUI-3) channel models. In the second technique, a high rate LDPC code is used in a concatenated coding structure as an outer code with a convolutional code as an inner code. The Convolutional codes are decode using two techniques bit-based maximum a posteriori probability (Log-MAP) decoder with its soft outputs feed into a binary LDPC decoder, and a symbol-based Log-MAP decoder with its soft outputs feed into a non-binary Galois Field LDPC decoder. The performance of such LDPC-CC concatenated codes is compared with the commonly used concatenated convolutional Reed-Solomon codes over the standard SUI-3 channel model, and the LDPC-CC codes showed better performance.
\end{abstract}

Keywords: LDPC, WiMAX, IEEE 802.16, LDPC codes, Concatenated Coding, Symbol based Log-MAP.

\section{INTRODUCTION}

The LDPC codes were originally invented and investigated by Gallager [1], and then recently investigated again in [2-6]. Gallager introduced an iterative decoding algorithm for decoding the LDPC codes, named the message passing algorithm. LDPC codes with the iterative decoding are considered serious competitors to turbo codes in terms of performance and complexity.

The serial concatenation of Reed-Solomon codes with convolutional codes is well known in channel coding. ReedSolomon codes are block codes that are good for correcting burst errors. Convolutional codes are good for correcting random errors. Together, the combination effectively corrects most errors caused by the wireless channel. Commonly, the convolutional codes are decoded to the maximum likelihood codeword and this is then used as input to the ReedSolomon decoder. Information is not used effectively in this process since the convolutional decoder used in this process returns a hard decision. This is due to that most ReedSolomon decoders are not capable of utilizing soft information. However, convolutional codes with soft-input softoutput decoder have shown to perform well in concatenated coding schemes [7].

In this paper replacing the Reed-Solomon codes of RSCC concatenated codes with high rate low density parity check codes is investigated. This has been proposed before

\footnotetext{
*Address correspondence to these authors at the Cairo University, Dept. of Electronics and Communications, Giza, Egypt, Tel: +1(662)915-1059; Fax: +1(662)915-7231; E-mail: kelmahgoub@ieee.org

Nile University, Wireless Intelligent Networks Center, Giza, Egypt; Tel: +202-3534-2146; Fax: +202-3534-1155;

E-mail:mnafie@nileuniversity.edu.eg
}

in [8], but no details were given on the decoding process, and the concatenated scheme was dealt with as one big code rather than a concatenation of two constituent codes. In this paper the technique of using bit-based Log-MAP and symbol-based Log- MAP [9-12] for decoding the convolutional code with its soft outputs feed into LDPC binary or nonbinary decoder is proposed. These inputs will be used to initialize the probabilities of various bits or symbols that are then used in the iterative LDPC decoding algorithm.

Note that using LDPC codes instead of RS has two extra advantages besides that the LDPC codes are decoded with soft-inputs. First the LDPC codes can be generated with any block length and any code rate. Moreover, LDPC codes outperform RS in many types of channels like the Binary Symmetric Channel (BSC), AWGN and 16-arry channels [8]. One of the techniques that can be used to generate high rate codes is to puncture lower rate codes to obtain the desired rate [9]. Hence, puncturing techniques of the LDPC codes proposed in the IEEE 802.16 standard $[13,14]$ are used to get higher rate LDPC codes for the concatenation system proposed [15-18].

One way of improving LDPC codes is making codes in which the variable nodes are grouped together into metavariables consisting of number of binary variables, and the check nodes are similarly grouped together into meta-checks. As before, a sparse graph can be constructed connecting meta-variables to meta-checks, with some freedom of the details of how the variables and checks within are wired up. One way to set the wiring is to work in a finite field, $G F(q)$, such as $G F(4)$ or $G F(8)$, and define low-density parity-check matrices using elements of $G F(q)$, and translate our binary messages into $G F(q)$ using a mapping such as the one for $G F(4)$ in [19]. Now, when messages are passed during decoding, those messages are probabilities and likelihoods over 
conjunctions of binary variables. For example if each group contains three binary variables then the likelihoods will describe the likelihoods of the eight alternative states of those bits. With carefully optimized constructions, the resulting codes over $G F(4), G F(8)$, and $G F(16)$ were shown to perform nearly one decibel better than comparable binary LDPC codes [12]. Hence, in this paper a parity check matrix for use over $G F(4)$ that is based on the structure presented in the IEEE 802.16 standard is presented.

This paper is organized as follows, in the next section novel parity check matrix over $G F(4)$ is proposed, and simulation results are provided to prove that the proposed code outperforms the IEEE 802.16 codes, over both AWGN and SUI-3 channel models [20]. In section III, the concatenated coding scheme are presented, and results showing the simulation results of the proposed scheme when binary and $G F(4)$ LDPC codes are used as the outer codes and both bit-based and symbol-based Log-MAP are used as convolutional decoders. Again the results prove that the proposed code outperforms the code in the IEEE standard. Section VI, provides a discussion of the new approaches and conclusion.

\section{LDPC CODES OVER GF(4) IN THE IEEE 802.16 PHYSICAL LAYER}

\section{A. Proposed Parity Check Matrix}

To design the parity check matrix over $G F(4)$, it is based on the parity check matrices proposed for binary LDPC in the IEEE 802.16 standard. The reason is that these codes were shown to have very good performance. Moreover, using the same structure as the binary codes might allow for some hardware reuse between the binary and our proposed $G F(4)$ codes. One of the advantages of the codes proposed in the standard is its versatility, as the code is defined with several block sizes according to an expansion factor $\left(\mathrm{z}_{f}\right)$. Moreover the codes have parity check matrices that are lower triangular allowing the encoding to be done in an efficient way without the need to first obtain the generator matrices. The standard LDPC codes also have several rates, namely $(1 / 2,2 / 3,3 / 4,5 / 6)[14]$.

In this section the same parity check matrix for the binary code is used, but instead of having only 1's in the matrix elements from $G F(4)$ are used, denoted by 1,2 and 3 . Hence, the non-zero entries of the matrix will be replaced by elements of $G F(4)$. Note that the greater the entropy of the syndrome the closer to the Shannon limit an optimal decoder can get [21, 22]. Hence, to find the best construction of the parity check matrix, a construction technique that maximizes the entropy of the syndrome under a particular channel model is used. The binary symmetric channel is used for making the calculations of the entropy feasible. For each distinct choice of $l$ non-zero entries in a row of the parity check matrix the marginal entropy of one element of the syndrome vector is examined and the non-zero elements that maximize the entropy are chosen. Then the created code will have its rows drawn from these choices. Note that the parity check matrix for the rate $1 / 2$ LDPC of the IEEE 802.16 standard has some rows with six non-zero entries, and other rows with seven non-zero entries.

The entropy of the syndrome for various combinations of the six, or seven, elements when chosen from $G F(4)$ is calcu- lated. The entropies of all the possibilities are shown in Figs. (1 and 2). It turned out that the vector which maximizes the entropy in case of six non-zero entries is $\left.\begin{array}{llllll}1 & 1 & 2 & 2 & 3 & 3\end{array}\right\}$, where 1,2 and 3 are the elements of $G F(4)$. In the case of seven non-zero entries the three vectors $\left\{\begin{array}{lllllll}1 & 1 & 1 & 2 & 2 & 3 & 3\end{array}\right\},\left\{\begin{array}{lll}1 & 1\end{array}\right.$

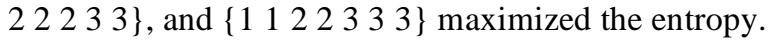

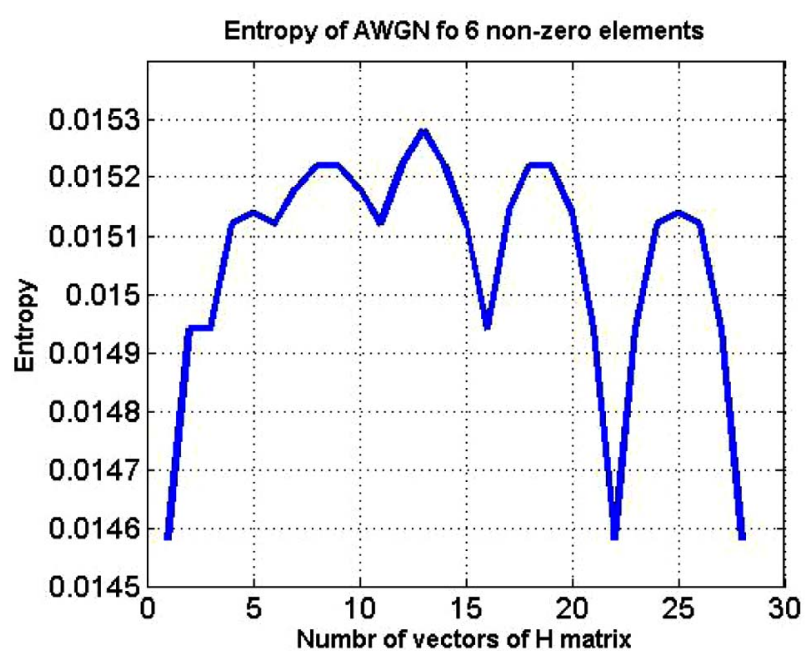

Fig. (1). Entropy over AWGN for 6 non-zero elements with probability of error $10^{-5}$.

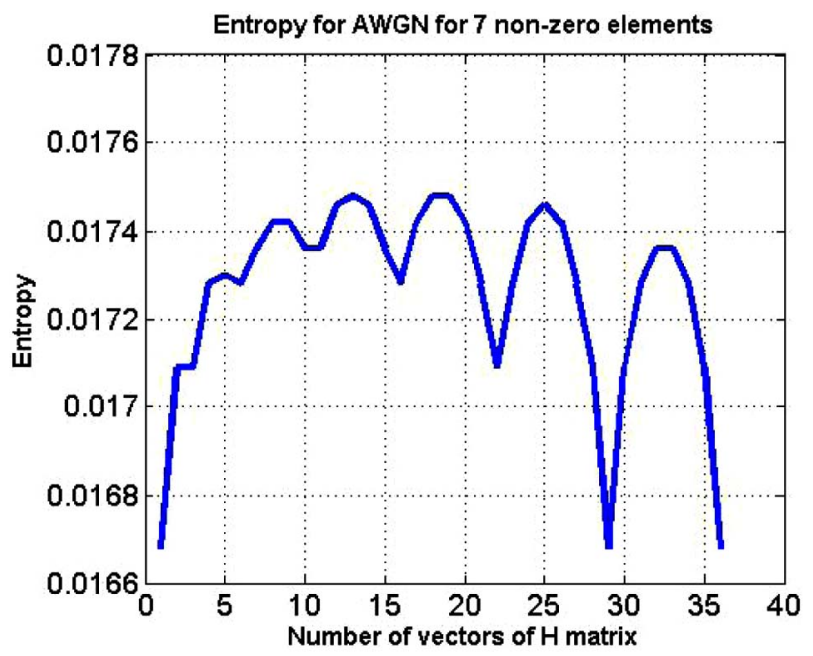

Fig. (2). Entropy over AWGN for 7 non-zero elements with probability of error $10^{-5}$.

Note that our results in Figs. $(\mathbf{1}$ and $\mathbf{2})$ are shown for a certain probability of error of the binary symmetric channel, but these findings were also verified at other probabilities of error as well. The optimization here is feasible since the number of non-zero entries in the original parity check matrix is only 6 or 7 . When the number of non-zero entries increase, the brute force optimization we have utilized will no longer be feasible and search for other optimization techniques should be implemented. Hence, the following construction is used. Starting the first non-zero entry with $\mathrm{a}=1$ and the next non-zero entry $b=2$ is used, and for the third non-zero entry $\mathrm{c}=3$ is used, and then using $\mathrm{a}=1$ again and so on. Note that this construction seems to be extendable to any $G F(\mathrm{q})$ by using all elements of $G F(\mathrm{q})$ before going back to 1 . However this was not verified. 


\section{B. Simulation Results}

The proposed LDPC code is simulated over both the AWGN channel and SUI-3 channel model and the results are compared with the binary LDPC codes proposed in the IEEE 802.16 standard, using the same block length and code rate of the codes used in the standard. Fig. (3) shows the bit error rate (BER) of the two rate $1 / 2$ codes over an AWGN channel model. Both codes use an input word with length 576 bits, and hence a 1152 bit output codeword. It is clear from the figure that our proposed code outperforms the original code especially at the high end of the simulated SNRs. The performance enhancement at $10^{-5} \mathrm{BER}$ is around $1 \mathrm{~dB}$.

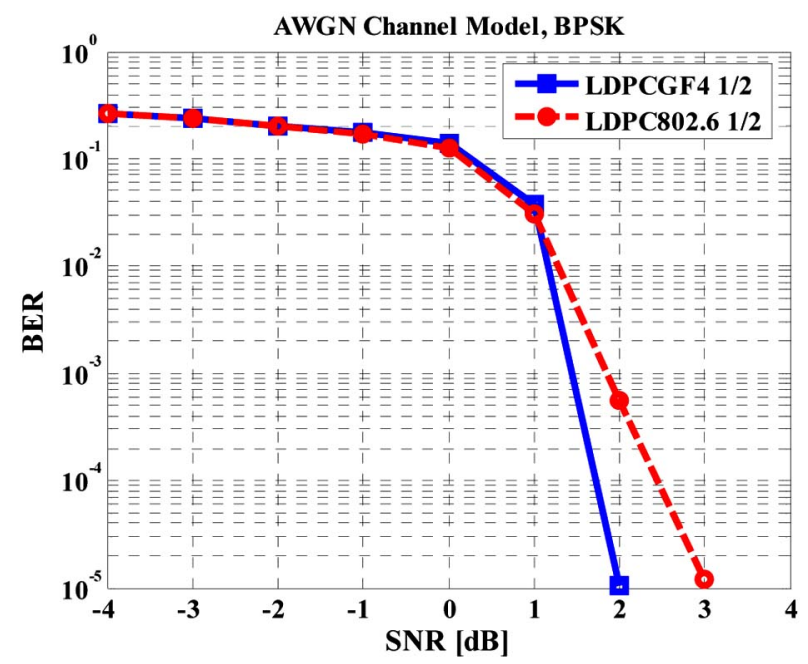

Fig. (3). Bit Error Rate for the rate $1 / 2$ LDPC code of the IEEE 802.16 standard compared with the proposed $G F(4)$ code over AWGN.

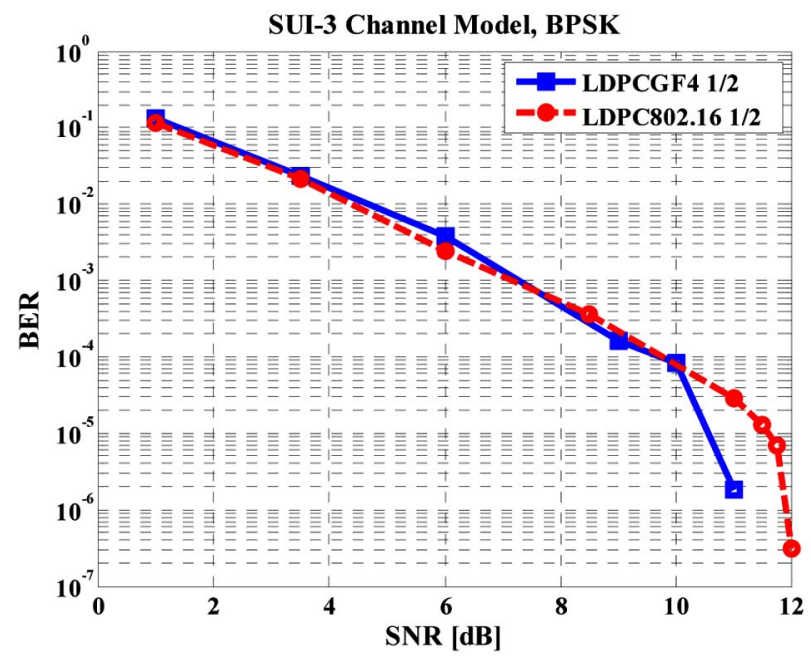

Fig. (4). Bit Error Rate for the rate $1 / 2$ LDPC code of the IEEE 802.16 standard compared with the proposed $G F(4)$ code over SUI3 channel model.

In Addition, the code was simulated over the SUI-3 channel model. Fig. (4) shows the BER of the 2 codes over SUI-3 channel model. Again, one can notice that the proposed code outperforms the code in the standard especially at high SNRs. Figs. (5 and $\mathbf{6}$ ) show the results of a rate $5 / 6$ $G F(4)$ code based on the binary rate $5 / 6$ LDPC code in the 802.16 standard over both AWGN and SUI-3 channel mod- els. Again, our constructed code outperforms the standard code over both channel models. These codes will be used later in the paper in the proposed concatenated scheme. The codeword length for both codes is 1152 bits.

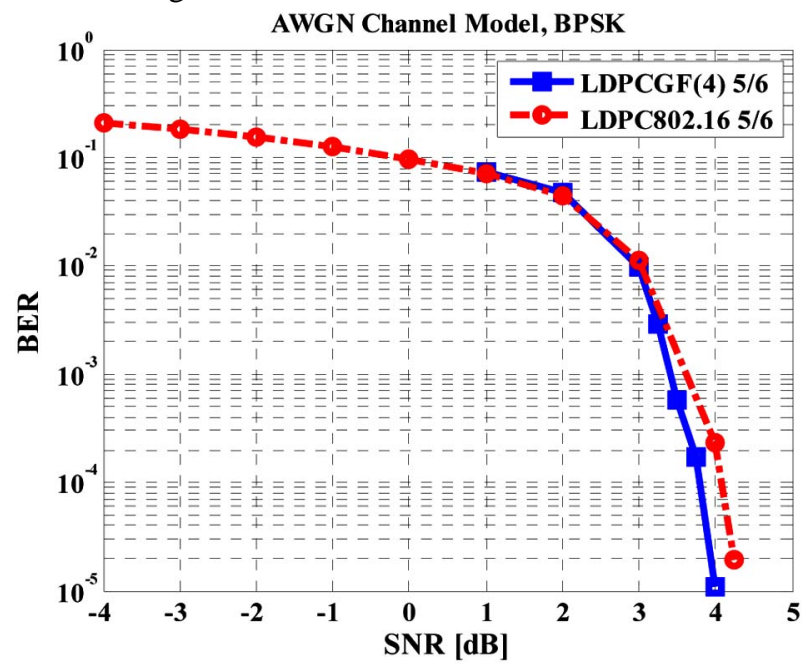

Fig. (5). Bit Error Rate for LDPC of IEEE 802.16 rate $5 / 6$ using both binary and $G F(4)$ with the proposed matrix over AWGN.

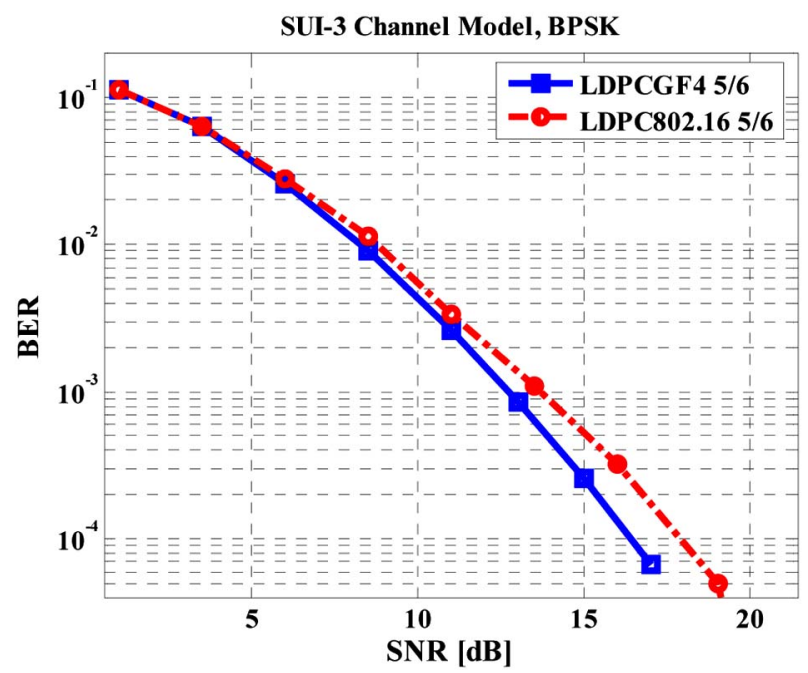

Fig. (6). Bit Error Rate for LDPC of IEEE 802.16 rate $5 / 6$ using both binary and $G F(4)$ with the proposed matrix over SUI-3 channel model.

\section{CONCATENATED LDPC - CC CODES}

\section{A. Proposed Concatenation System}

In this scheme, the LDPC code is used as the outer code, similar to the way the RS codes are used in the well known concatenated schemes. The convolutional code is used as the inner code, as shown in Fig. (7). The soft output bit-based and symbol-based Log-MAP algorithms are used in decoding the convolutional code. The Log-MAP algorithm produces likelihood ratios, or probabilities, for various bits or symbols that were used as an input to the convolutional encoder. The Log-MAP algorithm is used since it is known to outperform other soft output convolutional decoding schemes, such as the soft output Viterbi algorithm (SOVA), or the Max-Log-MAP. One has to note, however, that if this system is to be implemented, a trade-off has to be achieved 
between performance and complexity, since, although the Log-MAP outperforms other decoding schemes, it has higher complexity and its performance might also suffer when implemented in fixed point architectures.

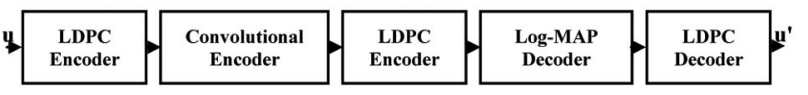

Fig. (7). Block diagram of the concatenated scheme.

The output of the Log-MAP decoding block is then passed as an input to the LDPC decoding block which utilizes the message passing algorithm described in section III. The LDPC decoding block then produces an estimate of the information stream. In case the outer code is $G F(\mathrm{q})$ LDPC code rather than a binary one and bit-based Log-MAP is used, the convolutional decoder is still assumed to output binary probabilities, which are then grouped together to obtain the probabilities of the various symbols over $G F(\mathrm{q})$. Furthermore, in case the outer code is $G F(\mathrm{q})$ LDPC code and symbol-based Log-MAP is used, this allows us to directly use the symbol likelihoods of the convolutional decoder as an input for the LDPC Galois Field decoder without any further re-grouping of the bits.

\section{B. Simulation Results with Bit-Based Log-MAP}

The proposed system is simulated over the SUI-3 channel model and it is compared with the code used in the IEEE 802.16. For the sake of a fair comparison, the same codeword length for the various coding options is used. Fig. (8) shows the BER of a rate $5 / 6$ LDPC concatenated with a rate $1 / 2$ convolutional code, the BER of a rate $7 / 8$ LDPC concatenated with a rate $1 / 2$ convolutional code, where the rate $7 / 8$ LDPC code is obtained by puncturing the rate $5 / 6$ LDPC code of the IEEE 802.16 standard. It compares these 2 codes to the BER of the rate $1 / 2$ convolutional code, the BER of a concatenated RS-CC code scheme and the rate $1 / 2$ LDPC. The codeword length of these codes is 1152 bits except for the rate $7 / 8$ code where it is 1096 . Again, all the codes are as specified in the IEEE 802.16 standard. All codes are simulated over SUI-3 channel model. Both the concatenated codes outperform the rate $1 / 2$ LDPC code used in the IEEE

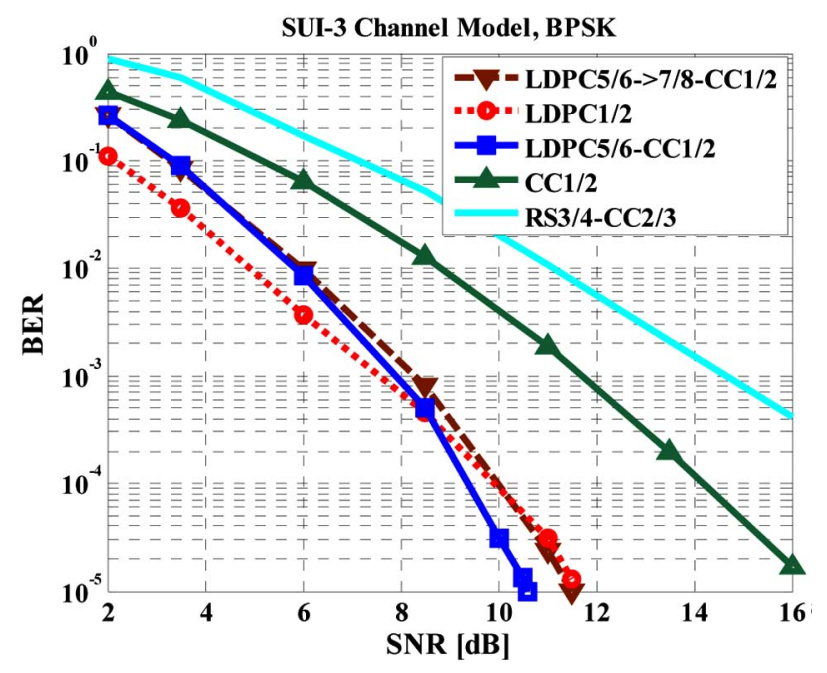

Fig. (8). Bit Error Rate of some LDPC-CC concatenated codes over SUI-3 channel model.
802.16 standard. Note that the concatenated code utilizing the rate $7 / 8$ code obtains performance that is close to that utilizing the rate $5 / 6$ code, although the puncturing pattern used to puncture the rate $5 / 6$ code was chosen at random. Optimizing this puncturing pattern, perhaps using similar schemes to the ones in [9] might even lead to better results. Fig. (9) shows the BER of a rate $5 / 6 G F(4)$ LDPC concatenated with a rate $1 / 2$ convolutional code over SUI-3 channel model.

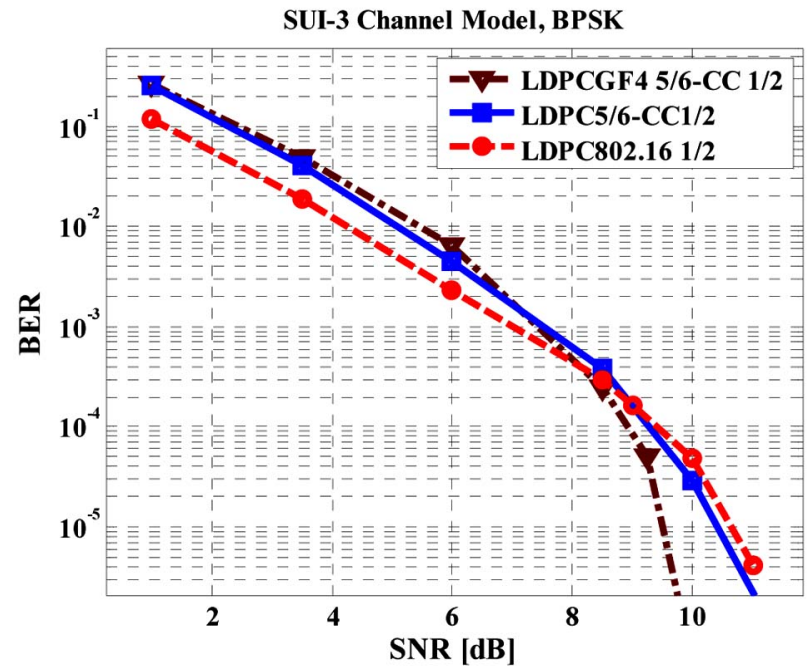

Fig. (9). Bit Error Rate of concatenated $G F(4)$ LDPC-CC over SUI3 channel model.

The curve clearly shows that this code outperforms the similar binary code, as well as the rate $1 / 2$ LDPC code. The performance enhancement is slightly higher than $1 \mathrm{~dB}$ at BER below $10^{-5}$. Note that the codeword length for these codes is 2304 bits.

\section{Simulation Results with symbol-based Log-MAP}

The proposed system over the AWGN and SUI-3 channel models is compared with the LDPC binary code used in IEEE802.16 and the LDPC-CC code using bit based LogMAP decoding. All the codes were simulated using 16QAM modulation and all the other blocks used in the physical layer WirelessMAN-OFDM PHY (Randomizer, FFT, and IFFT). For the sake of a fair comparison, Almost same codeword length for the various coding options is used.

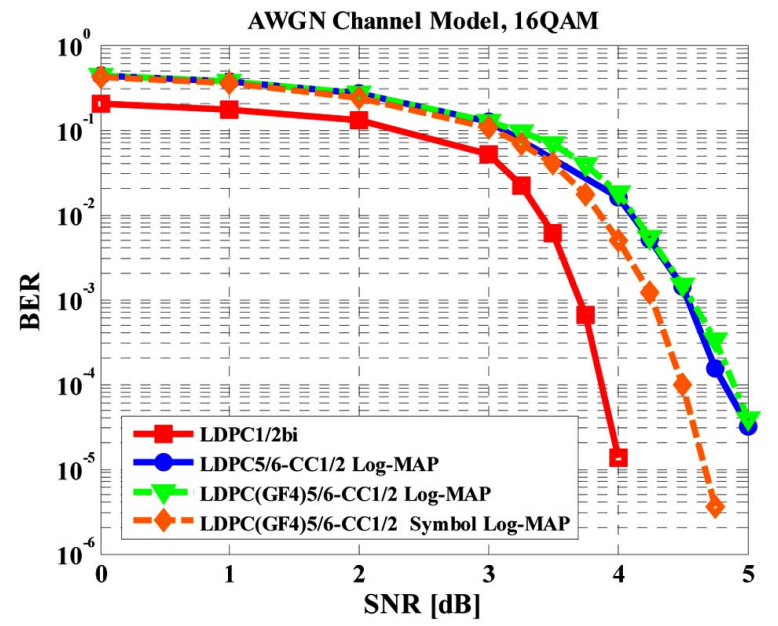

Fig. (10). Bit Error Rate of some LDPC and LDPC-CC concatenated codes over AWGN channel model. 
Fig. (10) shows the BER of code 1 (rate $1 / 2$ LDPC binary code used in WiMAX), the BER of code 2 (rate $5 / 6$ LDPC concatenated with a rate $1 / 2$ convolutional code using bit based Log-MAP), the BER of code 3 (rate $5 / 6$ LDPC GF(4) concatenated with a rate $1 / 2$ convolutional code using bit based Log-MAP) and the BER of code 4 (rate $5 / 6$ LDPC $G F(4)$ concatenated with a rate $1 / 2$ convolutional code using Symbol based Log-MAP). The codeword length of code 1 is 870 bits, for code 2, 3 and 4 the codeword length is 960 bits. It can be noticed that code 4 outperformance both code 2 and 3 which implies that using the symbol based Log-MAP decoding improves the performance of the LDPC-CC concatenated code.

Fig. (11) shows the BER of code 1, 2, 3, and 4 over SUI3 channel model. It can be noticed that code 4 outperforms both code 2 and 3, which shows that using symbol based Log-MAP improves the performance of LDPC-CC concatenated code. Also the LDPC-CC codes outperform the LDPC code as single code especially at higher Signal to Noise (SNR) ratios.

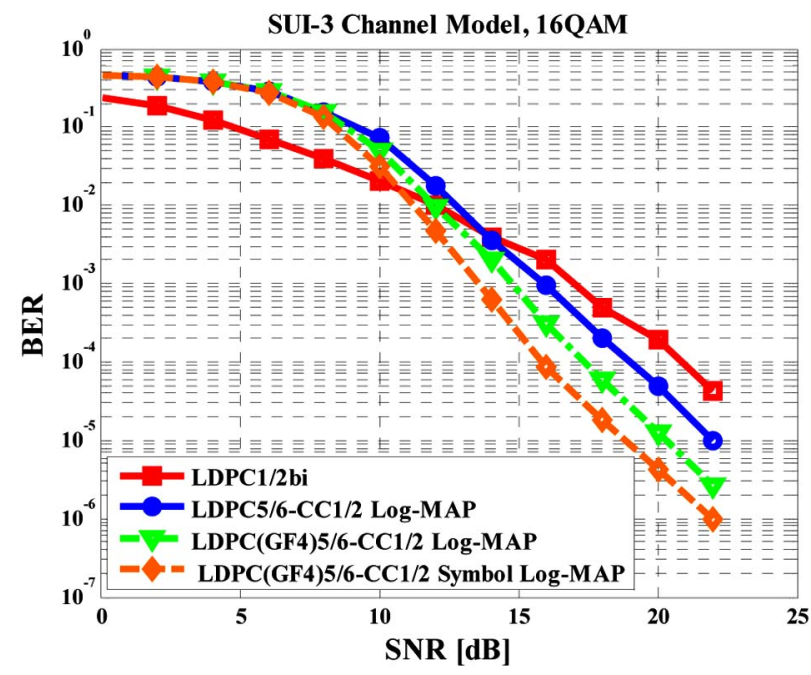

Fig. (11). Bit Error Rate of some LDPC and LDPC-CC concatenated codes over SUI-3 channel model.

\section{CONCLUSION}

In this paper, a new $G F(4)$ LDPC code where its parity check matrix is based on the parity check matrix of the binary LDPC code of the IEEE 802.16 standard is proposed. The proposed code outperforms the binary variant for the rate $1 / 2$ and rate $5 / 6$ codes for both the AWGN and SUI-3 channel models. By choosing the entries to maximize the entropy the performance of the LDPC code is enhanced. Note that the entries of the parity check matrix were optimized for the rate $1 / 2$ cases and for the binary symmetric channel model (works for AWGN). Hence, one would expect that further enhancement could be obtained if the optimization can be carried out over the proper channel model (SUI-3 for example).

In addition, a novel concatenated coding scheme using LDPC code as an outer code and convolutional code as an inner code is proposed. The coding scheme outperforms both LDPC and convolutional code used in IEEE 802.16 as single codes. Also, the code outperforms the concatenated RS-CC code. We also showed that using an LDPC code over $G F(4)$ in the concatenated code architecture leads to further enhancement in performance. In addition, it was showed that using symbol-based Log-MAP outperforms using bit-based Log-Map in such concatenated structure over both AWGN and SUI-3 channel models for the 16 QAM.

From the results of the $G F(4)$ presented, the authors suggest that LDPC codes over $G F(\mathrm{q})$ in general can be used instead of binary LDPC code in both single and concatenated coding structures. Using LDPC codes over $G F(8)$ or $G F(16)$ for example should lead to even better performance, However, caution must be taken in choosing the non-zero elements of the parity check matrices. In an adaptive system the non-zero entries can be changed according to the changes in the channel to always maximize the entropy. How to achieve this in a computationally feasible way is still an open issue. In addition, using symbol based interleaver between the LDPC code and the CC in the concatenated structure might enhance the performance further.

\section{REFERENCES}

[1] R. G. Gallager, "Low density parity check codes", IRE Trans. Info. Theory, IT-8: pp. 21-28, 1962.

[2] D. J. C. MacKay and R. M. Neal, "Near Shannon Limit Performance of Low Density Parity Check Codes", Electronics Letters, vol. 32, no. 18, pp. 1645-1646, 1996. Reprinted Electronics Letters, vol. 33, no. 6, pp. 457- 458, 1997.

[3] T. J. Richardson and R. L. Urbanke, "Efficient Encoding of LowDensity Parity - Check Codes," IEEE Trans. Info. Theory, vol. 47, no. $2,2001$.

[4] T. Hehn, J.B. Huber, Laendner, "Improved iterative decoding of LDPC codes from the IEEE WiMAX standard," In: Proceeding of International ITG conference on Source and Channel Coding, 2010.

[5] A. A. Ovchinnikov, A. Belogolovy, and E. Krouk, "Iterative Decoding of Concatenated Low-Density Parity-Check Codes," Patent application number: 20090249163.

[6] D. Declercq and M. Fossorier, "Decoding Algorithms for Nonbinary LDPC Codes over GF(q)," IEEE Trans. Commun.., vol. 55, no.4, pp. 633-643, 2007.

[7] R. Johannesson and K. Zigangirov, Fundamentals of Convolutional Coding, IEEE, 1998

[8] E. A. Ratzer, "Intersected Low-Density Parity- Check and Convolutional Codes" [Online]. Available: http://www.inference.phy.cam.ac.uk/ear23/papers/

[9] B. Vucetic and J. Yuan, Turbo Codes Principles and Applications, Kluwer Academic publishers group, 2000.

[10] S. Papaharalabos, P. Sweeney and B.G. Evans, "Constant LogMAP Decoding Algorithm for Duo-Binary Turbo Codes," Electronics Letters, vol. 42, no. 12, pp.709-710, 2006.

[11] D. J. C. MacKay and M. C. Davey "Evaluation of Gallager codes for short block length and high rate applications," In Codes, Systems and Graphical Models, Ed. By Marcus and J. Rosenthal, volume 123 of IMA Volumes in Mathematics and its Applications, pp. 113-130.

[12] H. Pishro-Nik and F. Fekri, "Results on Punctured LDPC Codes," Proceedings IEEE Information Theory Workshop (ITW), pp. 215$219,2004$.

[13] IEEE (802.16d) Standard 2004 [Online]. Available: http://www.standards.ieee.org/wireless/

[14] IEEE P802.16e/D10 Standard, 2005 [Online]. Available: http://www.standards.ieee.org/wireless/

[15] K. ElMahgoub and M. Nafie, "Concatenation and Puncturing of Low Density Parity Check Codes," ICENCO2006, Cairo, Egypt, pp.18-22, 2006.

[16] K. ElMahgoub and M. Nafie, "On the Enhancement of LDPC Codes in the IEEE 802.16 Physical Layer," ICC 2007 proceedings, pp. 683-688, 2007. 
[17] K. ElMahgoub and M. Nafie, "Symbol Based Log-MAP in Concatenated LDPC Convolutional codes," IEEE CCNC proceedings, 2010 .

[18] Khaled ElMahgoub and Mohammed Nafie, Enhancements to Low Density Parity Check Codes, LAP Lambert Academic Publishing, 2010

[19] D. J. C. MacKay, Information Theory, Inference, and Learning Algorithms, Version 7.0 (third printing). Cambridge University Press 2003.
[20] Vinko Erceg "SUI Channel Models for IEEE 802.16," IEEE 802.16.3c-01/29r4 [Online]. Available: www.ieee802.org/16/tg3/ contrib/802163c-01_29r4.pdf

[21] Matthew C. Davey and David J. C. Mackay "Low Density Parity Check Codes over GF(q), "IEEE Communications Letters, vol. 2, pp. $165-167,1998$.

[22] D. J. C. Mackay, "Optimizing sparse graph codes over $G F(\mathrm{q})$," [Online]. Available: http://www.inference.phy.cam.ac.uk/mackay/ CodesGallager.html

Received: March 21, 2011

(C) ElMahgoub and Nafie; Licensee Bentham Open.

This is an open access article licensed under the terms of the Creative Commons Attribution Non-Commercial License (http://creativecommons.org/licenses/by-nc/3.0/) which permits unrestricted, non-commercial use, distribution and reproduction in any medium, provided the work is properly cited. 